\title{
El uso de la gamificación en la enseñanza del emprendimiento: una revisión de la literatura
}

\author{
Martí-Parreño, J. ${ }^{\text {a }}$; Seguí-Mas, D. ${ }^{\text {' }}$ \& Seguí-Mas, E. \\ ${ }^{\mathrm{a}, \mathrm{b}}$ Universidad Europea de Valencia , ${ }^{\mathrm{c}}$ Universitat Politècnica de València
}

\begin{abstract}
Resumen
El uso de juegos serios (serious games), y de la gamificación en general, ha irrumpido con fuerza en el ámbito educativo en todos sus niveles, desde la educación primaria hasta la educación superior. Ejemplos de este uso incluye juegos serios para la enseñanza de idiomas, matemáticas, física o comercio internacional, por poner algunos ejemplos. El aprendizaje del emprendimiento no ha sido ajeno a este proceso y la literatura académica da prueba de numerosos ejemplos de juegos serios utilizados para la enseñanza del emprendimiento. No obstante, a pesar del creciente uso de la gamificación en el contexto del emprendimiento, los trabajos académicos que han analizado en profundidad este cuerpo creciente de literatura académica son todavía escasos si no inexistentes. Por ello, el objetivo principal de este trabajo de investigación es analizar en profundidad la literatura académica del uso de la gamificación en el contexto del emprendimiento. Para ello se ha realizado una revisión en profundidad de documentos cientificos con el objetivo de: a) conocer mejor las metodologías empleadas en el estudio de este tema, b) analizar las muestras empleadas por los investigadores, c) identificar los diferentes niveles educativos en los que se está desarrollando esta investigación, y d) identificar el ámbito geográfico de esta investigación. Los principales resultados de este análisis han permitido identificar algunas tendencias en este tema de estudio asi como señalar algunas lagunas en la investigación que ofrecen nuevas oportunidades para los investigadores.
\end{abstract}

Palabras clave: gamificación, juegos serios, serious games, emprendimiento, revisión de literatura 


\section{Introducción}

El uso de juegos serios utilizados para desarrollar valores, actitudes y habilidades relacionadas con el emprendimiento tiene ya un largo recorrido que supera los 40 años de historia. La literatura académica muestra numerosos ejemplos con importantes instituciones educativas como Harvard y Carnegie-Mellon entre las pioneras en desarrollar juegos serios para enseñar emprendimiento. Así, podemos remontarnos a los años 70 para encontrar las primeras experiencias bien documentadas sobre el uso de simulaciones y juegos relacionados con el mundo de los negocios y el emprendimiento. Baldwin (1974) narra los titánicos esfuerzos por introducir el Carnegie-Mellon University Simulation y el Harvard Business Game en sus cursos (incluida la petición de ayuda a una base de las Fuerzas Aéreas norteamericanas primero, y a la compañía Mosanto después, para poder usar el juego ya que los equipos informáticos del campus de su universidad no disponían de memoria de procesamiento suficiente para hacer funcionar la simulación). Ese mismo año aparece un nuevo juego, STARTING A SMALL BUSINESS (Gupta \& Hamman, 1974), que será seguido en la década de los 80 , por nuevos juegos para la enseñanza del emprendimiento: ENTREPRENEUR: A simulation (Smith \& Golden 1987), ENTREPRENURIAL SIMULATION PROGRAM (Penderghast, 1988) (para una revisión de estos tres juegos ver: Wolfe \& Bruton (1994). Tras varias décadas de experimentación con el uso de los juegos como metodología para enseñar emprendimiento aparecen las primeras revisiones bibliográficas sobre el tema (Faria, 2001) sugiriendo que el área de estudio empieza a consolidarse. En la actualidad, los juegos serios para enseñar emprendimiento se cuentan por decenas e incluyen una amplia variedad de posibilidades que se dirigen desde a los adolescentes (Hot Shot Business) hasta estudiantes de secundaria y universidad (Industry Player, Innov8) e incluso profesionales (GoVenture Small Business', Market Place, Sim Venture, The Enterprise Game). También existen juegos destinados específicamente a estudiantes de escuelas de negocios (MetaVals, The Balance Sheet Game). La dificultad de estos juegos también varía pudiéndose encontrar juegos de un nivel básico que pueden ser jugados hasta por niños de primaria (Team Up). Existen otros juegos que exigen un nivel de conocimientos más avanzados (Virtonomics) (para una revisión de estos juegos ver: Bellotti (2014). La mayoría de estos juegos pueden ser jugados online vía la web. Es el caso de Entrepreneurship Simulation: The Startup Game desarrollado por la Wharton School de la Universidad de Pennsylvania.

El incremento constante en la aparición de nuevos juegos serios para enseñar el emprendimiento está motivado, entre otros factores, por las diferentes políticas que están alentando la enseñanza del emprendimiento como una vía de desarrollo y bienestar social. En este sentido, tanto la OECD (2013) como la Comisión Europea (2006, 2013) han señalado la importancia de la educación en emprendimiento por el papel que juega no sólo en desarrollar competencias, atributos y actitudes en los jóvenes sino también en su forma 
de pensar (Williams, 2015). De hecho, en los últimos años la Comisión Europea ha financiado diversos proyectos para la enseñanza del emprendimiento mediante juegos serios (PLAYER, StartUp_EU, ENTRExplorer). Unido al esfuerzo de las políticas que están promoviendo la enseñanza del emprendimiento en todos los ámbitos y etapas educativas, la investigación académica también debe incrementar su esfuerzo con el objetivo de analizar rigurosamente el impacto de los diferentes proyectos y acciones que se están desarrollando. En este sentido, este trabajo académico pretende contribuir al área de conocimiento mediante una revisión en profundidad de documentos científicos sobre gamificación y emprendimiento con el objetivo de: a) conocer mejor las metodologías empleadas en el estudio de este tema, b) analizar las muestras empleadas por los investigadores, c) identificar los diferentes niveles educativos en los que se está desarrollando esta investigación, y d) identificar el ámbito geográfico de esta investigación. Para ello se han planteado las siguientes cuestiones de investigación (CIs):

CI1: ¿Qué metodologías están empleando los investigadores en el estudio de este tema?

CI2: ¿Qué muestras (tipo y tamaño) están empleando los investigadores?

CI3: ¿En qué niveles educativos se está desarrollando esta investigación?

CI4: ¿En qué ámbito geográfico se está desarrollando esta investigación?

Los principales resultados de este análisis no sólo permitirán identificar algunas tendencias en este tema de estudio sino señalar algunas lagunas en la investigación que ofrecen nuevas oportunidades para los investigadores.

\section{Metodología}

La metodología empelada en este estudio es una revisión comprensiva de la literatura. Mediante la revisión de un número de estudios sobre un mismo tema, la revisión en profundidad de literatura académica persigue establecer reglas generales y/o paradigmas basados en afirmaciones concluyentes encontradas en la literatura académica (Pasadeos, Phelps, \& Kim, 1998).

\section{Procedimiento, estrategia de búsqueda y muestra}

La recopilación de documentos para el análisis se realizó durante octubre de 2017, y con el fin de recopilar datos de años completos, el límite para los años de análisis se estableció en 2016. Se utilizó la siguiente estrategia de búsqueda: TS= ((GAMIFICATION OR "EDUCATIONAL GAMES" OR "GAME-BASED LEARNING" OR "SERIOUS GAMES") AND (ENTREPRENEUR*)). Con esta estrategia de búsqueda, se recuperaron 
un total de 59 documentos. Los investigadores leyeron cuidadosamente los títulos y resúmenes para verificar que todos los documentos recuperados coincidieran con los criterios para ser incluidos en el análisis. Una vez realizado este proceso de revisión, la muestra final de análisis consistió en 24 documentos. La tabla 1 recoge la información bibliométrica básica de los documentos analizados.

Tabla 1. Documentos analizados

\begin{tabular}{|c|c|c|c|c|}
\hline Autor & Año & Título & $\begin{array}{c}\text { Tipo de } \\
\text { documento }\end{array}$ & Fuente \\
\hline $\begin{array}{l}\text { Garcia-Fernandez, J ; } \\
\text { Fernandez-Gavira, J ; } \\
\text { Sanchez-Oliver, AJ ; } \\
\text { Grimaldi-Puyana, M }\end{array}$ & 2017 & $\begin{array}{l}\text { Gamificación y } \\
\text { aplicaciones } \\
\text { móviles para } \\
\text { emprender: una } \\
\text { propuesta } \\
\text { educativa en la } \\
\text { enseñanza superior }\end{array}$ & Artículo & $\begin{array}{c}\text { IJERI- } \\
\text { INTERNATIONAL } \\
\text { JOURNAL OF } \\
\text { EDUCATIONAL } \\
\text { RESEARCH AND } \\
\text { INNOVATION }\end{array}$ \\
\hline $\begin{array}{l}\text { Tsalapatas, } \mathrm{H} ; \\
\text { Heidmann, O; } \\
\text { Houstis, E }\end{array}$ & 2017 & $\begin{array}{l}\text { A Serious Game } \\
\text { for Digital Skill } \\
\text { Building Among } \\
\text { Individuals at } \\
\text { Risk, Promoting } \\
\text { Employability and } \\
\text { Social Inclusion }\end{array}$ & $\begin{array}{l}\text { Actas de } \\
\text { Congreso }\end{array}$ & $\begin{array}{l}\text { Lecture Notes of } \\
\text { the Institute for } \\
\text { Computer Sciences, } \\
\text { Social Informatics } \\
\text { and } \\
\text { Telecommunication } \\
\text { s Engineering }\end{array}$ \\
\hline $\begin{array}{c}\text { Rachid Lamrani, El } \\
\text { Hassan Abdelwahed, } \\
\text { Souad Chraibi, } \\
\text { Sara Qassimi, Meriem } \\
\text { Hafidi and Abdelaziz } \\
\text { El Amrani }\end{array}$ & 2017 & $\begin{array}{l}\text { Serious Game to } \\
\text { Enhance and } \\
\text { Promote Youth } \\
\text { Entrepreneurship }\end{array}$ & $\begin{array}{l}\text { Actas de } \\
\text { Congreso }\end{array}$ & $\begin{array}{c}\text { EUROPE AND } \\
\text { MENA } \\
\text { COOPERATION } \\
\text { ADVANCES IN } \\
\text { INFORMATION AND } \\
\text { COMMUNICATION } \\
\text { TECHNOLOGIES }\end{array}$ \\
\hline Pinto, $\mathrm{R}$ & 2016 & $\begin{array}{c}\text { Empreendedorism } \\
\text { o e gamificação no } \\
\text { desenvolvimento } \\
\text { profissional }\end{array}$ & Artículo & LINHA D AGUA \\
\hline $\begin{array}{c}\text { Bellotti, F.; Berta, R.; } \\
\text { De Gloria, A.; } \\
\text { Lavagnino, E.; }\end{array}$ & 2013 & $\begin{array}{l}\text { A Gamified Short } \\
\text { Course for } \\
\text { Promoting }\end{array}$ & $\begin{array}{l}\text { Actas de } \\
\text { Congreso }\end{array}$ & $\begin{array}{l}\text { 13th IEEE Annual } \\
\text { International } \\
\text { Conference on }\end{array}$ \\
\hline
\end{tabular}




\begin{tabular}{|c|c|c|c|c|}
\hline $\begin{array}{c}\text { Dagnino, F.; } \\
\text { Antonaci, A.; Ott, M }\end{array}$ & & $\begin{array}{l}\text { Entrepreneurship } \\
\text { among ICT } \\
\text { Engineering } \\
\text { Students }\end{array}$ & & $\begin{array}{l}\text { Advanced Learning } \\
\text { Technologies } \\
\text { (ICALT) }\end{array}$ \\
\hline $\begin{array}{l}\text { Allegra, M; Fulantelli, } \\
\text { G.; Gentile, M.; La } \\
\text { Guardia,D.; Taibi,D. ; } \\
\text { Zangara, G. }\end{array}$ & 2010 & $\begin{array}{l}\text { An agent-based } \\
\text { serious game for } \\
\text { entrepreneurship }\end{array}$ & $\begin{array}{l}\text { Actas de } \\
\text { Congreso }\end{array}$ & $\begin{array}{l}\text { PROCEEDINGS OF } \\
\text { THE 5TH } \\
\text { INTERNATIONAL } \\
\text { CONFERENCE ON } \\
\text { VIRTUAL } \\
\text { LEARNING, ICVL } \\
2010\end{array}$ \\
\hline Remmele, B & 2010 & $\begin{array}{l}\text { Game-Based } \\
\text { Fostering of } \\
\text { Entrepreneurial } \\
\text { Attitudes? }\end{array}$ & $\begin{array}{l}\text { Actas de } \\
\text { Congreso }\end{array}$ & $\begin{array}{l}\text { 4th European } \\
\text { Conference on } \\
\text { Games-Based } \\
\text { Learning (ECGBL) }\end{array}$ \\
\hline $\begin{array}{c}\text { Allegra, M; Fulantelli, } \\
\text { G.; Gentile, M.; D.; } \\
\text { Taibi,D. }\end{array}$ & 2009 & $\begin{array}{c}\text { On line } \\
\text { environments to } \\
\text { enhance } \\
\text { entrepreneurial } \\
\text { mindsets in young } \\
\text { students }\end{array}$ & $\begin{array}{l}\text { Actas de } \\
\text { Congreso }\end{array}$ & $\begin{array}{c}\text { ICVL } 2009 \text { - } \\
\text { PROCEEDINGS OF } \\
\text { THE 4TH } \\
\text { INTERNATIONAL } \\
\text { CONFERENCE ON } \\
\text { VIRTUAL LEARNING }\end{array}$ \\
\hline $\begin{array}{c}\text { Fonseca, B., } \\
\text { Morgado, L., Paredes, } \\
\text { H., Martins, P., } \\
\text { Gonçalves, R., Neves, } \\
\text { P., ... \& Sanders, R. }\end{array}$ & 2012 & $\begin{array}{c}\text { PLAYER - a } \\
\text { European Project } \\
\text { and a Game to } \\
\text { Foster } \\
\text { Entrepreneurship } \\
\text { Education for } \\
\text { Young People }\end{array}$ & Artículo & $\begin{array}{l}\text { JOURNAL OF } \\
\text { UNIVERSAL } \\
\text { COMPUTER } \\
\text { SCIENCE }\end{array}$ \\
\hline Starčič, A. I & 2008 & $\begin{array}{l}\text { Simulation game- } \\
\text { based virtual } \\
\text { learning }\end{array}$ & $\begin{array}{l}\text { Actas de } \\
\text { Congreso }\end{array}$ & $\begin{array}{c}\text { 8th WSEAS } \\
\text { International } \\
\text { Conference on } \\
\text { Distance Learning } \\
\text { and Web } \\
\text { Engineering } \\
\end{array}$ \\
\hline $\begin{array}{c}\text { Protopsaltis, A., } \\
\text { Borotis, S., Connolly, } \\
\text { T., \& Hainey, T. }\end{array}$ & 2014 & $\begin{array}{c}\text { Teaching } \\
\text { Entrepreneuship } \\
\text { Using Serious } \\
\text { Games in a Web }\end{array}$ & $\begin{array}{l}\text { Actas de } \\
\text { Congreso }\end{array}$ & $\begin{array}{l}2014 \text { International } \\
\text { Conference on } \\
\text { Interactive Mobile } \\
\text { Communication }\end{array}$ \\
\hline
\end{tabular}




\begin{tabular}{|c|c|c|c|c|}
\hline & & 2.0 environment & & $\begin{array}{l}\text { Technologies and } \\
\text { Learning (IMCL) }\end{array}$ \\
\hline Tak-Ming, L. & 2008 & $\begin{array}{l}\text { The Effect of User } \\
\text { Experience } \\
\text { Measurement on } \\
\text { Entrepreneurship } \\
\text { Business Venture } \\
\text { Simulation Game } \\
\text { Design }\end{array}$ & $\begin{array}{l}\text { Actas de } \\
\text { Congreso }\end{array}$ & $\begin{array}{l}\text { 2nd European } \\
\text { Conference on } \\
\text { Games-Based } \\
\text { Learning }\end{array}$ \\
\hline $\begin{array}{l}\text { Denk, N., Röhsner, } \\
\text { U., Ertl, B., } \\
\text { Wernbacher, T., \& } \\
\text { Pfeiffer, A }\end{array}$ & 2016 & $\begin{array}{l}\text { A Social Network } \\
\text { Game for } \\
\text { Encouraging Girls } \\
\text { to Engage in ICT } \\
\text { and } \\
\text { Entrepreneurship: } \\
\text { Findings of the } \\
\text { Project MIT-MUT }\end{array}$ & $\begin{array}{l}\text { Actas de } \\
\text { Congreso }\end{array}$ & $\begin{array}{l}\text { PROCEEDINGS OF } \\
\text { THE 3RD } \\
\text { EUROPEAN } \\
\text { CONFERENCE ON } \\
\text { SOCIAL MEDIA }\end{array}$ \\
\hline $\begin{array}{c}\text { Newbery, R., Lean, J., } \\
\text { \& Moizer, J. }\end{array}$ & 2016 & $\begin{array}{l}\text { Evaluating the } \\
\text { impact of serious } \\
\text { games: the effect } \\
\text { of gaming on } \\
\text { entrepreneurial } \\
\text { intent }\end{array}$ & Artículo & $\begin{array}{l}\text { INFORMATION } \\
\text { TECHNOLOGY \& } \\
\text { PEOPLE }\end{array}$ \\
\hline $\begin{array}{l}\text { Heath, C. D., Baron, } \\
\text { T., Gary, K., \& } \\
\text { Amresh, A. }\end{array}$ & 2016 & $\begin{array}{c}\text { Reflection on } \\
\text { Assumptions from } \\
\text { Designing Female- } \\
\text { Centric } \\
\text { Educational } \\
\text { Games }\end{array}$ & $\begin{array}{l}\text { Actas de } \\
\text { Congreso }\end{array}$ & $\begin{array}{l}\text { SERIOUS GAMES, } \\
\text { JCSG 2016, 2nd } \\
\text { International Joint } \\
\text { Conference on } \\
\text { Serious Games } \\
\text { (JCSG) }\end{array}$ \\
\hline $\begin{array}{c}\text { Damani, B., } \\
\text { Sardeshpande, V., \& } \\
\text { Gaitonde, U. }\end{array}$ & 2015 & $\begin{array}{c}\text { Use of serious } \\
\text { games for creating } \\
\text { awareness about } \\
\text { social enterprises }\end{array}$ & Artículo & $\begin{array}{l}\text { JOURNAL OF } \\
\text { COMPUTERS IN } \\
\text { EDUCATION }\end{array}$ \\
\hline $\begin{array}{c}\text { Lameras, P., Tsiatsos, } \\
\text { T., Petridis, P., Tolis, } \\
\text { D., Liarokapis, F., }\end{array}$ & 2015 & $\begin{array}{c}\text { Creative Thinking } \\
\text { Experimentations } \\
\text { for }\end{array}$ & $\begin{array}{l}\text { Actas de } \\
\text { Congreso }\end{array}$ & $\begin{array}{c}\text { PROCEEDINGS OF } \\
2015 \\
\text { INTERNATIONAL } \\
\text { CONFERENCE ON }\end{array}$ \\
\hline
\end{tabular}




\begin{tabular}{|c|c|c|c|c|}
\hline $\begin{array}{c}\text { Anastasiadou, D., ... } \\
\text { \& Arnab, S. }\end{array}$ & & $\begin{array}{c}\text { Entrepreneurship } \\
\text { with A Disruptive, } \\
\text { Personalised and } \\
\text { Mobile Game- } \\
\text { based Learning } \\
\text { Ecosystem }\end{array}$ & & $\begin{array}{c}\text { INTERACTIVE } \\
\text { MOBILE } \\
\text { COMMUNICATION } \\
\text { TECHNOLOGIES } \\
\text { AND LEARNING } \\
\text { (IMCL) }\end{array}$ \\
\hline Bontchev, B. & 2015 & $\begin{array}{l}\text { Customizable } 3 \mathrm{~d} \\
\text { video games as } \\
\text { educational } \\
\text { software }\end{array}$ & $\begin{array}{l}\text { Actas de } \\
\text { Congreso }\end{array}$ & $\begin{array}{l}\text { 7th International } \\
\text { Conference on } \\
\text { Education and New } \\
\text { Learning } \\
\text { Technologies } \\
\text { (EDULEARN) }\end{array}$ \\
\hline $\begin{array}{l}\text { Dagnino, F. M., } \\
\text { Antonaci, A., Ott, M., } \\
\text { Lavagnino, E., } \\
\text { Bellotti, F., Berta, R., } \\
\text { \& De Gloria, A. }\end{array}$ & 2015 & $\begin{array}{l}\text { The eSG Project: } \\
\text { A Blended } \\
\text { Learning Model } \\
\text { for Teaching } \\
\text { Entrepreneurship } \\
\text { Through Serious } \\
\text { Games }\end{array}$ & $\begin{array}{l}\text { Actas de } \\
\text { Congreso }\end{array}$ & $\begin{array}{l}\text { 10th European } \\
\text { Conference on } \\
\text { Innovation and } \\
\text { Entrepreneurship } \\
\text { (ECIE) }\end{array}$ \\
\hline $\begin{array}{l}\text { La Guardia, D., } \\
\text { Gentile, M., Dal } \\
\text { Grande, V., } \\
\text { Ottaviano, S., \& } \\
\text { Allegra, M. }\end{array}$ & 2014 & $\begin{array}{l}\text { A Game Based } \\
\text { Learning Model } \\
\text { for } \\
\text { Entrepreneurship } \\
\text { Education }\end{array}$ & $\begin{array}{l}\text { Actas de } \\
\text { Congreso }\end{array}$ & $\begin{array}{c}\text { 4th World } \\
\text { Conference on } \\
\text { Learning, Teaching } \\
\text { and Educational } \\
\text { Leadership } \\
\text { (WCLTA) }\end{array}$ \\
\hline $\begin{array}{c}\text { Protopsaltis, A., } \\
\text { Hainey, T., Borosis, } \\
\text { S., Connolly, T., } \\
\text { Copado, J., \& Hezner, } \\
\text { S. }\end{array}$ & 2013 & $\begin{array}{c}\text { StartUp_EU: } \\
\text { Using Game- } \\
\text { Based Learning } \\
\text { and Web } 2.0 \\
\text { Technologies to } \\
\text { Teach } \\
\text { Entrepreneurship } \\
\text { to Secondary } \\
\text { Education } \\
\text { Students }\end{array}$ & $\begin{array}{l}\text { Actas de } \\
\text { Congreso }\end{array}$ & $\begin{array}{l}\text { 7th European } \\
\text { Conference on } \\
\text { Games Based } \\
\text { Learning }\end{array}$ \\
\hline Sernack, J. L. & 2013 & $\begin{array}{c}\text { Applying the } \\
\text { Disruptive Israeli } \\
\text { Innovation Model }\end{array}$ & $\begin{array}{l}\text { Actas de } \\
\text { Congreso }\end{array}$ & $\begin{array}{l}\text { 8th European } \\
\text { Conference on } \\
\text { Innovation and }\end{array}$ \\
\hline
\end{tabular}




\begin{tabular}{|c|c|c|c|c|}
\hline & & $\begin{array}{l}\text { to Re-Inventing } \\
\text { Corporate } \\
\text { Education }\end{array}$ & & $\begin{array}{l}\text { Entrepreneurship } \\
\text { (ECIE) }\end{array}$ \\
\hline $\begin{array}{l}\text { Bellotti, F., Berta, R., } \\
\text { De Gloria, A., } \\
\text { Lavagnino, E., } \\
\text { Dagnino, F., Ott, M., } \\
\text {... \& Mayer, I. S. }\end{array}$ & 2012 & $\begin{array}{c}\text { Designing a } \\
\text { Course for } \\
\text { Stimulating Entrep } \\
\text { reneurship in } \\
\text { Higher Education } \\
\text { through Serious } \\
\text { Games }\end{array}$ & $\begin{array}{l}\text { Actas de } \\
\text { Congreso }\end{array}$ & $\begin{array}{c}\text { 4th International } \\
\text { Conference on } \\
\text { Games and Virtual } \\
\text { Worlds for Serious } \\
\text { Applications (VS- } \\
\text { GAMES) }\end{array}$ \\
\hline $\begin{array}{l}\text { Logofatu, B., Columb, } \\
\text { C., \& Dumitrache, A }\end{array}$ & 2011 & $\begin{array}{c}\text { Game based } \\
\text { learning in open } \\
\text { distance learning }\end{array}$ & $\begin{array}{l}\text { Actas de } \\
\text { Congreso }\end{array}$ & $\begin{array}{l}\text { 7th International } \\
\text { Scientific } \\
\text { Conference } \\
\text { eLearning and } \\
\text { Software for } \\
\text { Education } \\
\end{array}$ \\
\hline
\end{tabular}

\section{Resultados}

La cuestión de investigación 1 tenía como objetivo responder a qué metodologías están empleando los investigadores en el estudio de este tema. El análisis de la muestra revela que un $41,67 \%$ de los estudios son teóricos, un $25 \%$ utilizan una aproximación cuantitativa, un $20,83 \%$ una aproximación cualitativa y el $12,50 \%$ restante utilizan un enfoque mixto cuantitativo/cualitativo. Con respecto a la cuestión de investigación 2 (¿Qué muestras (tipo y tamaño) están empleando los investigadores?), los resultados reflejan que el tipo de muestra predominante es la de estudiantes universitarios (principalmente de grado, con sólo 2 investigaciones estudiando alumnos de máster y 1 estudio utilizando estudiantes ya graduados). Tan sólo uno de los estudios utiliza una muestra de estudiantes de formación profesional. En cuanto a los tamaños muestrales son bastante heterogéneos, con muestras que oscilan entre los 20 y los 263 casos. La cuestión de investigación 3 tenía como objetivo conocer en qué niveles educativos se está desarrollando esta investigación. El 45,83\% de los estudios se han desarrollado en el contexto de la educación superior, mientras que el $8,33 \%$ lo han hecho en la educación secundaria. Un $25 \%$ de los estudios no aluden a un nivel educativo específico mientras que un $16,67 \%$ de los trabajos se dirige a "jóvenes" en general y uno de los estudios hace referencia a "escolares" sin especificar nivel educativo. Finalmente, la cuestión de investigación 4 tenía como objetivo conocer mejor en qué ámbito geográfico se está desarrollando esta investigación. Atendiendo a la filiación de los 
investigadores, encontramos 19 países en los que se ha desarrollado investigación sobre el tema. Italia ocupa un papel destacado en los estudios analizados representando un 17,95\% de la muestra, seguida de Alemania y Reino Unido (12,82\%) y Bélgica y España (7,69\%).

\section{Conclusiones}

Esta revisión de la literatura académica sobre el uso de la gamificación en la enseñanza del emprendimiento parece sugerir que nos encontramos ante un área de estudio incipiente tal y como parece reflejar el hecho de que casi la mitad de los documentos analizados sean de carácter teórico. El hecho de que el tipo de muestra predominante sea de estudiantes universitarios parece evidenciar la importancia que se le está dando a formar a los estudiantes universitarios en competencias emprendedoras con el objetivo de incrementar sus posibilidades de empleabilidad una vez finalizados sus estudios. El predominio de países europeos en los estudios analizados parece justificarse por el impulso dado desde la Comisión Europea a través de la financiación de proyectos de enseñanza del emprendimiento a través de los juegos (tal y como queda reflejado en los agradecimientos de los estudios analizados).

\section{Agradecimientos}

Esta investigación ha sido financiada por Laureate International Universities a través del David A. Wilson Award for Excellence in Teaching and Learning bajo la beca LIUWIL2015.

\section{Referencias}

Baldwin, L. E. (1974). Using complex simulations in policy courses in institutions with limited resources. En Developments in Business Simulation and Experiential Learning: Proceedings of the Annual ABSEL conference (Vol. 1), 186-189.

Bellotti, F., Berta, R., De Gloria, A., Lavagnino, E., Antonaci, A., Dagnino, F., Ott, M., Romero M., \& Mayer, I. S. (2014). Serious games and the development of an entrepreneurial mindset in higher education engineering students. Entertainment Computing, 5(4), 357-366.

Commission of the European Communities. (2005). Implementing the Community Lisbon Programme: Fostering Entrepreneurial Mindsets Through Education and Learning: Communication from the Commission to the Council, the European Parliament, the European 
Economic and Social Committee and the Committee of the Regions. Office for Official Publications of the European Communities.

European Commission. (2013). Entrepreneurship 2020 action plan: reigniting the entrepreneurial spirit in Europe.

Faria, A. J. (2001). The changing nature of business simulation/gaming research: A brief history. Simulation \& Gaming, 32(1), 97-110.

Gupta, S. K., \& Hamman, R. T. (1974). Starting a small business: player's manual. Prentice Hall.

OECD (2013). Culture: the role of entrepreneurship education in Entrepreneurship at a Glance 2013, OECD Publishing.

Pasadeos, Y., Phelps, J., \& Kim, B. H. (1998). Disciplinary impact of advertising scholars: Temporal comparisons of influential authors, works and research networks. Journal of Advertising, 27(4), $53-70$.

Penderghast, T.F. (1988). Entrepreneur Simulation Program. Sandiego, CA: Harcourt Brace Jovanovich.

Smith, J. R., \& Golden, P. A. (1987). Entrepreneur: A simulation. Boston: Houghton Mifflin College Division.

Williams, D. (2015). The impact of SimVenture on the development of entrepreneurial skills in management students. Industry and Higher Education, 29(5), 379-395.

Wolfe, J., \& Bruton, G. (1994). On the use of computerized simulations for entrepreneurship education. Simulation \& Gaming, 25(3), 402-415. 